\section{Some Aspects of the Lur'e Problem}

M. A. L. THATHACHAR AND M. D. SRINATH, MEMBER, IEEE

Abstract-The problem of absolute stability of feedback systems containing a single nonlinearity is considered for the case of the linear transfer function having an equal number of finite poles and zeros. Explicit Liapunov functions are presented and frequencydomain criteria are derived for systems for which the nonlinear function $f(\cdot)$ belongs to the class $A_{\infty}(f$ lies in the first and third quadrants) or its subclasses such as monotonically increasing functions $\left(f \in M_{\infty}\right)$, odd-monotonic functions $\left(f \in 0_{\infty}\right)$, and functions with a power-law restriction $\left(f \in P_{\infty}\right)$. A new class of functions with restricted asymmetry having the property $\mid f(0) / f(-0), \leq c$ for all $\theta$ $(\theta \neq 0)$ is introduced, and the results obtained can be used to establish stability in some cases even when the Nyquist plot of the linear part transfer function lies in all the four quadrants and the nonlinearity is not necessarily odd. Restrictions on the derivative of the nonlinearity have been taken into account by means of a transformation, and the resulting stability criterion is seen to be an improvement over those obtained in some earlier papers.

\section{INTRODUCTION}

The Lur'e problem and its modifications have attracted considerable attention in recent years. The results of Popov [1] have been extended and frequency-domain criteria presented for determining asymptotic stability in the large (ASIL) of systems consisting of a linear transfer function with nonlinear feedback [2]-[6]. Many of these publications [4]-[6] treat the case where the degree of the denominator polynomial of the linear part transfer function is at least one higher than that of the numerator. For $f \in A_{\infty}$ even the results of Brockett and Willems [3] are applicable only to this case. Recent papers by $\mathrm{Ku}$ and Chieh [7] and Johnson [8] have considered the case of $f \in A_{\infty}$ and linear transfer function being the ratio of equal-order polynomials; the derivation is rather involved in the former, and neither paper presents an explicit Liapunov function.

This paper derives stability criteria for equal-order systems with nonlinear feedback and gives explicit Liapunov functions to prove ASIL. Nonlinearities belonging to several classes are considered, and stability criteria are presented that require the existence of a positive real function, which yields a positive real product when multiplied by the linear transfer function. The multiplier is permitted, in certain cases, to have complex conjugate poles and zeros and is thus more general than the type of multipliers in earlier results [3]. A new class of functions with restricted asymmetry is introduced, and the results for this case are used to prove stability in cases where earlier results fail.

These results, in conjunction with a transformation of the nonlinear function, are used to provide results for cases where the derivative of the nonlinearity is bounded, thus extending the results in earlier papers [4], [5] where no such bound was placed on the derivative.

\section{The SyStem}

The dynamical system considered in this paper is described by the vector-matrix equations

$$
\begin{aligned}
& \dot{x}=A x-b f(\sigma) \\
& \sigma=h^{T} x-\eta f(\sigma)
\end{aligned}
$$

where $x$ is an $n$ vector representing the state of the system; $b$ and $h$ are constant $n$ yectors. $A$ is a constant $n \times n$ matrix all of whose eigenvalues have negative real parts, $\sigma$ is a real-valued scalar time function, and $\eta$ is a non-negative scalar.

It is assumed that $f(\cdot)$ belongs to the class $A_{\infty}$ or its subclasses such as monotonically increasing functions $\left(f \in M_{\infty}\right)$, monotonic functions with restricted asymmetry $\left(f \in N_{\infty}^{c}\right)$, odd-monotonic func-

\footnotetext{
Manuscript received June 12, 1966; tevised February 6, 1967, and April 21 1967. The authors are with the Dept. of Electrical Engineering, Indian Institute of Science, Bangalore 12, India.
}

tions $\left(f \in 0_{x}\right)$, or odd-monotonic functions with a power-law restriction $\left(f \in P_{\infty}{ }^{m}\right)[3],[5]$.

The nonlinear function is assumed to be differentiable in order to ensure the continuity of the Liapunov function (introduced in the next section) and its first partials. It is further assumed that $1+\eta f^{\prime}(\sigma)$ $>0$, where $f^{\prime}(\sigma) \triangleq d f(\sigma) / d \sigma$, so that the mapping $x \rightarrow \sigma$ may be unique. It should be noted that this condition is automatically satisfied when $f(\cdot)$ is monotonically increasing.

It can easily be derived that the transfer function $G(s)$ relating the output $\sigma$ to the input $-f(\sigma)$ is given by

$$
G(s)=h^{T}(s I-A)^{-1} b+\eta=G_{1}(s)+\eta
$$

where $\eta$ is the value of $G(s)$ evaluated at $s=\infty$.

The results for $f \in A_{\infty}$ (the Popov case) are derived first and the derivation is indicated for the other cases.

\section{The Stability Criterion: Popoy Case}

Consider as a candidate for a Liapunov function

$$
V=\frac{1}{2} x^{T} P x+\beta_{0}\left[\int_{0}^{\sigma} f(\sigma) d \sigma+\frac{\eta}{2} f^{2}(\sigma)\right]
$$

with $P=P^{T}>0$ and $\beta_{0} \geq 0$. Using

$$
h^{T} \dot{x}=\left[1+\eta f^{\prime}(\sigma)\right] \dot{\sigma}
$$

which is obtained from (1), and adding and subtracting $\alpha f(\sigma) \sigma$ where $\alpha \geq 0$, the time derivative $\dot{V}$ along the trajectories of the system can be written as

$$
\begin{aligned}
\dot{V}= & \frac{1}{2} x^{T}\left(A^{T} P+P A\right) x-f(\sigma) x^{T}\left[P b-\beta_{0} A^{T} h-\alpha h\right] \\
& -\left[\beta_{0} h^{T} b+\alpha \eta\right] f^{2}(\sigma)-\alpha \sigma f(\sigma) .
\end{aligned}
$$

A straightforward application of the Meyer-Kalman-Yakubovich lemma [9], [4] then leads to the result that $\dot{V} \leq 0$ and $\dot{V}$ is not identically zero along a trajectory, provided

$$
\left(\beta_{0} h^{T} b+\alpha \eta\right)+\operatorname{Re}\left(\alpha+\beta_{0} A\right) h^{T}(j \omega I-A)^{-1} b \geq 0
$$

for all real $\omega$. The proof follows that in Meyer [9]. With some simplification of (6), the stability criterion can be stated as follows.

\section{Theorem 1}

The system (1) is ASIL for $f \in A_{\infty}$, satisfying $1+\eta f^{\prime}(\sigma)>0$, if there exist constants $\alpha>0$ and $\beta_{0} \geq 0$, such that

$$
\operatorname{Re} Z(j \omega) G(j \omega) \geq 0
$$

for all real $\omega$, where

$$
Z(s)=\alpha+\beta_{0} s .
$$

\section{Further Restrictions oN the Nonlinearity}

By placing more restrictions on the nonlinearity, stability can be predicted for a larger class of systems [2]-[6]. The results for equalorder transfer functions can be obtained by using the appropriate Liapunov function in [4], [5] with the additional term $\frac{1}{2} \beta_{0} \eta f^{2}(\sigma)$. Cases where the transfer function has a simple pole at the origin can be treated in a similar manner by considering the equations of indirect control [8].

As an example let $f \in M H_{\infty}$. Then $f(\cdot)$ satisfies

$$
\begin{aligned}
0<\frac{f(\theta)}{\theta} & <\infty ; \quad(\theta \neq 0) \\
f(0) & =0
\end{aligned}
$$

and

$$
0 \leq\left(\theta_{1}-\theta_{2}\right)\left[f\left(\theta_{1}\right)-f\left(\theta_{2}\right)\right]
$$

for all $\theta, \theta_{1}$, and $\theta_{2}$

Choose a Liapunov function of the form

$V=\frac{1}{2} x^{T} P x+\beta_{0}\left[\int_{0}^{\sigma} f(\sigma) d \sigma+\frac{\eta}{2} f^{2}(\sigma)\right]+\sum_{i} \beta_{i} \int_{0}^{h^{T} B_{i} x} f(\sigma) d \sigma$ 
where

$$
\begin{aligned}
P & =P^{T}>0 \\
B_{i} & =\frac{\gamma_{i}}{\beta_{i}}\left[\lambda_{i} I+A\right]^{-1}
\end{aligned}
$$

$\beta_{0}, \gamma_{i}, \beta_{i} \geq 0, i=1, \cdots, v$, where $v$ is an integer less than or equal to the number of real zeros of $G(s)$, and

$-\lambda_{i}$ is a real zero of $G(s), \lambda_{i} \neq 0$.

Adding and subtracting terms of the form

$$
\alpha \sigma f(\sigma)+\sum_{i} \gamma_{i}\left[f\left(h^{T} B_{i} x\right)-f(\sigma)\right]\left[h^{T} B_{i} x-\sigma\right]
$$

from $\dot{V}$ and following the approach in Narendra and Neuman [4], the stability criterion can be stated as follows.

\section{Theorem 2}

The system (1) is ASIL for $f \in M_{\infty}$ if there exist constants $\alpha>0$ and $\beta_{0}, \gamma_{i} \geq 0$, such that

1)

$$
\operatorname{Re} Z(j \omega) G(j \omega) \geq 0
$$

where

$$
Z(s)=\alpha+\beta_{0} s+\sum_{i} \gamma_{i} \frac{s+c_{i} \lambda_{i}}{s+\lambda_{i}} ; \quad 0 \leq c_{i} \leq 1
$$

2) $-\lambda_{i}$ is a nonzero real zero of $G(s)$.

It should be noted that the multiplier $Z(s)$ derived above is any RL driving-point impedance with its poles at the real zeros of $G(s)$.

The above result makes use of only the real zeros of $G(s)$, as the poles of $Z(s)$. By limiting the asymmetry of the nonlinear function, it is possible to use complex zeros of $G(s)$ in the multiplier.

For example, let $f \in N_{\infty}^{c}$. Then, in addition to $(9), f(\cdot)$ satisfies $|f(\theta) / f(-\theta)| \leq c,(\theta \neq 0)$, where $c \geq 1$.

If $-\lambda_{i} \pm j \mu_{i}$ and $-\lambda_{k} \pm j \mu_{k}$ are pairs of complex zeros of $G(s)$, define the following matrices:

$$
\begin{aligned}
& B_{i}=\frac{g_{i}}{\beta_{i}}\left[\left(\lambda_{i}-j \mu_{i}\right) I+A\right]^{-1} \\
& R_{i}=B_{i}+B_{i}^{*} ; \quad \mathscr{J}_{i}=j\left(B_{i}-B_{i}^{*}\right)
\end{aligned}
$$

where $q_{i}$ is a complex scalar and $\beta_{i}$ is a non-negative real number. Matrices are defined similarly for $k$-subscript terms.

Using a Liapunov function of the form

$$
\begin{aligned}
V(x)= & \frac{1}{2} x^{T} P x+\beta_{0} \int_{0}^{\sigma} f(\sigma) d \sigma+\frac{\beta_{0 \eta}}{2} f^{2}(\sigma) \\
& +\sum_{i=1}^{v_{1}}\left[\beta_{i} \int_{0}^{h^{T} \mathscr{G}_{i} x} f(\sigma) d \sigma+\beta_{i} \int_{0}^{h^{T} \mathfrak{I}_{i}^{x}} f(\sigma) d \sigma\right] \\
& +\sum_{k=v_{1}+1}^{v}\left[\beta_{k} \int_{0}^{h^{T} \mathfrak{I}_{k x}} f(\sigma) d \sigma+\beta_{k} \int_{0}^{h^{T} \mathfrak{J}_{k^{x}}} f(\sigma) d \sigma\right]
\end{aligned}
$$

and following the approach in Thathachar et al. [5], the results for $f \in N_{\infty}{ }^{c}$ can be stated as follow's.

\section{Thearem 3}

The system (1) is ASIL for $f \in N_{\infty}$ if there exist $\alpha>0$ and nonnegative constants $\beta_{0}, \beta_{i}, \beta_{i}{ }^{\prime}, \beta_{k}, \beta_{k}{ }^{\prime}, \alpha_{i}, \alpha_{i}{ }^{\prime}, \alpha_{k}, \alpha_{k}{ }^{\prime}$, and $\gamma_{i}, \gamma_{i}{ }^{\prime}, \gamma_{k}, \gamma_{k}{ }^{\prime}$ for $i=1, \cdots, v_{1}$ and $k=\left(v_{1}+1\right), \cdots, v$, where $i$ is an integer less than or equal to the number of zeros of $G(s)$, such that

$$
\text { 1) } \quad \operatorname{Re} Z(j \omega) G(j \omega) \geq 0
$$

for all real $\omega$, where

$$
\begin{aligned}
Z(s)= & \beta_{0} s+\left[\alpha+\sum_{i}\left(\gamma_{i}+\gamma_{i}^{\prime}\right)+\sum_{k}\left(\gamma_{k}+\gamma_{k}^{\prime}\right)\right] \\
& -\sum_{i}\left[\frac{\left(\gamma_{i}-j \gamma_{i}^{\prime}\right) q_{i} / \beta_{i}}{s+\lambda_{i}-j \mu_{i}}+\frac{\left(\gamma_{i}+j \gamma_{i}^{\prime}\right) q_{i}^{*} / \beta_{i}}{s+\lambda_{i}+j \mu_{i}}\right] \\
& +\sum_{k}\left[\frac{\left(\gamma_{k}-j \gamma_{k}^{\prime}\right) q_{k} / \beta_{k}}{s+\lambda_{k}-j \mu_{k}}+\frac{\left(\gamma_{k}+j \gamma_{k}\right) q_{k}^{*} / \beta_{k}}{s+\lambda_{k}+j \mu_{k}}\right] \\
\frac{q_{i}}{\beta_{i}}= & \frac{\gamma_{i}}{2 \beta_{i}}-j \frac{\gamma_{i}^{\prime}}{2 \beta_{i}^{\prime}}, \quad \frac{q_{k}}{\beta_{k}}=\frac{\gamma_{k}}{2 \beta_{k}}-j \frac{\gamma_{k}^{\prime}}{2 \beta_{k}^{\prime}}
\end{aligned}
$$

2)

$$
\begin{array}{ll}
\lambda_{i}=\frac{\gamma_{i}+\epsilon_{i}}{\beta_{i}}=\frac{\gamma_{i}^{\prime}+\epsilon_{i}^{\prime}}{\beta_{i}^{\prime}}, & \mu_{i}=\frac{\alpha_{i}}{\beta_{i}}=\frac{\alpha_{i}^{\prime}}{\beta_{i}^{\prime}} \\
\lambda_{k}=\frac{\gamma_{k}+\epsilon_{k}}{\beta_{k}}=\frac{\gamma_{k}^{\prime}+\epsilon_{k}^{\prime}}{\beta_{k}^{\prime}}, & \mu_{k}=\frac{\alpha_{k}}{\beta_{k}}=\frac{\alpha_{k}^{\prime}}{\beta_{k}^{\prime}}
\end{array}
$$

$-\lambda_{i} \pm j \mu_{i}$ and $-\lambda_{k} \pm j \mu_{k}$ being zeros of $G(s)$

3) $\epsilon_{i} \geq \max \left(\alpha_{i}, c \alpha_{i}{ }^{\prime}\right) ; \quad \epsilon_{i}^{\prime} \geq \max \left(\alpha_{i}, \alpha_{i}{ }^{\prime}\right)+(c-1) \gamma_{i}{ }^{\prime}$

$$
\epsilon_{k} \geq \max \left(\alpha_{k}, c \alpha_{k}{ }^{\prime}\right)+(c-1)_{\gamma_{k} ;} \epsilon_{k}^{\prime} \geq \max \left(\alpha_{k}, \alpha_{k}^{\prime}\right) \text {. }
$$

\section{Reduction to A SECTOR}

As indicated [10], use of a transformation along with the result for equal-order transfer functions permits extension to the finiterange case of the results of Popov, etc.

Let

$$
f_{1} \in A_{K} \quad \text { i.e., } 0<\sigma_{1} f_{1}\left(\sigma_{1}\right)<K \sigma_{\mathfrak{l}}{ }^{2} \text {. }
$$

Let

$$
\sigma=\sigma_{1}-f(\sigma) / K \text { and } f(\sigma)=f_{1}\left(\sigma_{1}\right) \text {. }
$$

Then

$$
\frac{f(\sigma)}{\sigma}=\frac{f_{1}\left(\sigma_{1}\right)}{\sigma_{1}-\frac{1}{K} f_{1}\left(\sigma_{1}\right)}=\frac{f_{1}\left(\sigma_{1}\right) / \sigma_{1}}{1-\frac{1}{K} \frac{f_{1}\left(\sigma_{1}\right)}{\sigma_{\mathrm{L}}}} .
$$

Hence $f_{1} \in A_{K}$ implies $f \in A_{\infty}$, and the system in Fig. 2 is zero-input equivalent to that in Fig. 1. Thus, the stability of the system in Fig. 1 with $f \in A_{\infty}$ implies that of the system in Fig. 2, with $f_{1} \in A_{K}$. Hence, the condition for ASIL of the system in Fig. 2 is given by

$$
\operatorname{Re}\left(\alpha+\beta_{0} s\right)\left[G_{1}(s)+\frac{1}{K}\right] \geq 0
$$

Now, from (21),

$$
\frac{d f(\sigma)}{d \sigma}=\frac{d f_{1}\left(\sigma_{1}\right) / d \sigma_{1}}{1-\frac{1}{K} \frac{d f_{1}\left(\sigma_{1}\right)}{d \sigma_{1}}} .
$$

Thus, if $f_{1}\left(\sigma_{1}\right)$ is monotonically increasing and $\left[d f_{1}\left(\sigma_{1}\right) / d \sigma_{1}\right]<K$, i.e., $f_{1} \in M_{K}, f(\sigma)$ is also monotonically increasing, i.e., $f \in M_{\infty}$. Conversely, if $f \in M_{*}, f_{1} \in M_{K}$.

Thus, the stability of the system in Fig. 2 with $f_{1} \in M_{K}$ is identical to that of the system in Fig. 1 with $f \in M_{\infty}$. Application of Theorem 2 then gives the condition for stability of $G_{1}(s)$ with nonlinear feedback $f_{1} \in M_{K}$.

\section{Theorem 4}

The dynamical system

$$
\begin{aligned}
\dot{x} & =A \dot{x}-b f_{1}\left(\sigma_{1}\right) \\
\sigma_{1} & =h^{T} x
\end{aligned}
$$

is ASIL for nonlinear feedback $f_{1} \in M_{K}$, under the conditions of Theorem 2, with $G_{1}(j \omega)+1 / K$ replacing $G(j \omega)$, where $G_{1}(s)$ $=h^{T}(s I-A)^{-1} b$. Similarly, for $f_{1} \in N_{K}^{c}$, i.e., $f \in N_{\infty}^{c}$ where $f$ is obtained from $f_{1}$ using (21), the criterion can be stated as follows.

\section{Theorem 5}

The system (25) is ASIL for $f_{1} \in N_{K}^{c}$ under the conditions of Theorem 3 with $G_{1}(j \omega)+1 / K$ replacing $G(j \omega)$.

Thus, by using the result for equal-order transfer functions along with a transformation, stability criteria can be stated for cases in which a maximum bound is placed on the derivative of the nonlinear function. The stability criteria presented elsewhere [4], [5] where no such restriction was placed on the slope of the nonlinearity, are of the form

$$
\operatorname{Re} Z(j \omega)\left[G_{1}(j \omega)+1 / K\right]-\alpha^{\prime} / K \geq 0
$$

where $\alpha^{\prime}$ is positive. Thus, it can be seen that the constant negative term $-\alpha^{\prime} / K$ has been eliminated in Theorems 4 and 5 ; hence, the conditions on the linear part have been relaxed. 


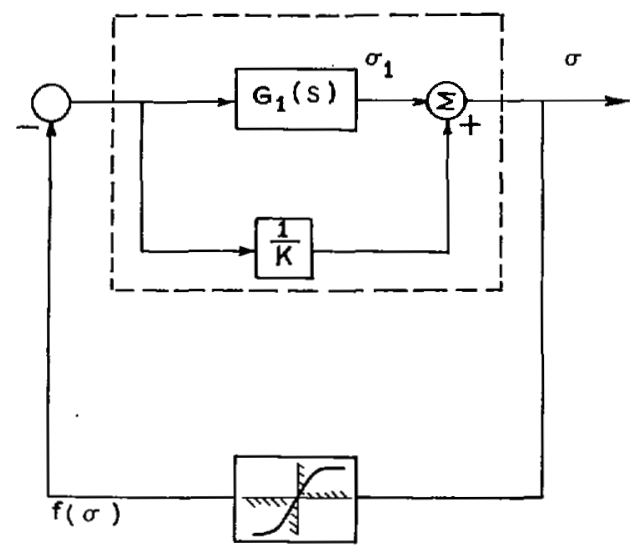

Fig. 1. Equal-order system with nonlinearity in the infinite sector.

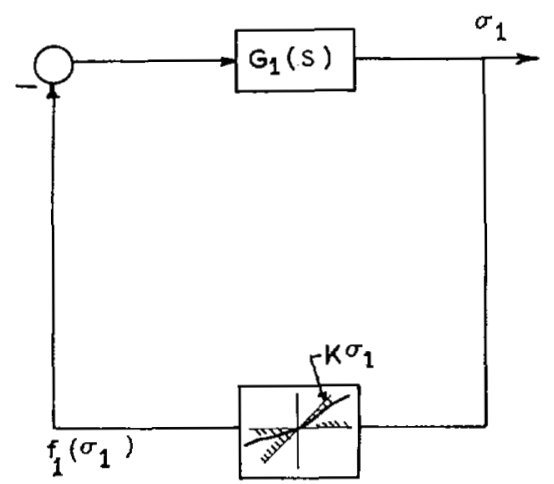

Fig. 2. Equivalent system with nonlinearity in a finite sector.

\section{Further Extexisions}

The stability properties of a system with $G(s)$ in the forward path and $f(\sigma)$ in the feedback path are the same as those of a system with $1 / G(s)$ in the forward path and $f^{-1}(\sigma)$ in the feedback path. When $f(\sigma)$ is in $M_{\infty} f^{-1}(\sigma)$ is also in $M_{x}$. Hence, when $\eta \neq 0$ and $f$ belongs to $H_{\infty}$ or its subclasses, one can replace $G(s)$ by $1 / G(s)$ in all the criteria derived in this paper. This applies to the finite sector case as well when $f_{1} \in M_{K}$. Even when $f$ is not invertible, using transformation (21) and the results in Aizerman and Gantmacher [11], $G(s)$ can be replaced by $1 / G(s)$.

From the above discussion, Theorem 6 follows.

\section{Theorem 6}

1) If $\eta \neq 0$, Theorems 1,2 , and 3 are valid even when $G(s)$ is replaced by $1 / G(s)$.

2) Theorems 4 and 5 remain valid even when $G_{1}(s)+1 / K$ is replaced by $\left[G_{1}(s)+1 / K\right]^{-1}$.

It may be noted that Theorem 6 implies that $[Z(s)]^{-1}$ can be used in the place of $Z(s)$ in all the previous theorems and is similar to the results obtained elsewhere [3], [6].

The criteria for the case of an odd monotonic nonlinearity can be obtained by setting $c=1$ in Theorems 3 and 5 . When the nonlinearity satisfies a power-law restriction $\left(f \in P_{\infty}{ }^{m}\right.$ or $\left.P_{K}{ }^{m}\right)$, results can be obtained by proceeding along the same lines as in Theorems
3 and 5 using appropriate inequalities given in Thathachar et al. [5]. The form of multiplier turns out to be the same as that found previously [5].

\section{EXAMPLE}

Consider the transfer function

$$
G(s)=\frac{\left(s^{2}+1.8 s+1\right)\left(s^{2}+4\right)}{s\left(s^{2}+2.1 s+1.2\right)(s+20)}
$$

with nonlinear feedback in $N_{\infty} c$.

The Nyquist plat of the transfer function lies in all the four quadrants; hence, the Popov criterion is not satisfied. Results of Narendra and Neuman [4] are not applicable here. Criteria in Brockett and Willems [3] cannot establish stability because $f$ is not odd. Even the results of O'Shea [6] applied after converting the problem to a finite sector problem, are not useful because no further assumptions such as boundedness are made on $f$; hence, stability cannot be established unless the phase angle of $G(j \omega)$ is in a $270^{\circ}$ band or $f$ is odd.

However, with the results in the present paper, one can chonse

$$
Z(s)=\frac{\left(s^{2}+2.1 s+1.2\right)(s+20)}{s^{2}+1.8 s+1}
$$

under the conditions of Theorem 3 with $c=2$, so that $Z(s) G(s)$ $=\left(s^{2}+4\right) / s$ is positive real. Thus the system is ASIL for all $f \in N_{x}{ }^{2}$

\section{Conclusions}

Explicit Liapunov functions are given for establishing asymptotic stability in the large of a feedback system with a single nonlinearity and a linear part with transfer function of the form of the ratio of equal-order polynomials. Stability criteria are presented for systems with various classes of nonlinearities, viz., $A_{\infty}, M_{\infty}$, and $N_{\infty} c$.

These results along with a transformation have been used to derive conditions for ASIL in the finite sector case. This permits extension of earlier results [4], [5] to the case of the nonlinearity with a restricted maximum value of the derivative. Thus, criteria are obtained for $f \in M_{K}, N_{K^{c}}$, etc. An example indicates that the present method can take care of situations where the other existing criteria fail. Though not explicitly stated, results can be obtained for the case of an equal-order transfer function, stable in a finite sector, by proceeding along the lines indicated in this paper.

\section{REFERENCES}

[1] V. M. Popov, "Absolute stability of nonlinear systems of automatic control," A utomalion and Remote Control, vol. 22, pt. 8. pp. 857-875, March 1962.

[2] G. Zames, "On the stability of nonlinear, time-varying feedback systems," Proc. Nat'l Electronics Conf . pp. 725-730, 1964

[3] R. W. Brockett and J. L. Willems "Frequency domain stability criteria," pt. I, IEEE Trans. A utomatic Control, vol. AC-10, pp. 255-261, July 1965; pt. 1I, ibid., pp. 407-413, October 1965 .

[4] K. S. Narendra and C. P. Neuman, "Stability ot a class of differential equations with a single monotone nonlinearity, " J. SIA M Control, vol. 4, no. 2, 1966. 5] M. A. L. Thathachar, M. D. Srinath, and H. K. Ramaptiyan, “On a modified Lur'e problem," IEEE Trans. A utomatic Control, vol. AC-12, December 1967.

[6] R. P. O'Shea, "A combined frequency-time domain stability criterion for

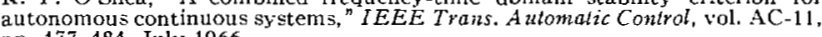
pp. $477-484$. July 1966.

[7] F. H. Ku and H. T. Chieh, "Extension of Popov's theoremsior stability of nonlinear control systems," J. Franklin Irst., vol. 279. pp. 401-416. June 1965.

8] C. D. Johnson, A note on control systems with one nonlinear element." IEEE Trans. Automatic Contral (Short Papers), vol. AC-11, pp. 122-124.

[9] K. R. Meyer: "On the existence of Liapunov functions for the problem of Lure." J.SIA A Control, vol. 3, no. 3. pp. 37.3-383, 1965.

[10] M. A. L. Thathachar, Mi. D. Srinath, and G. Krishna, "Stability with nonlinearity in a sector." IEEE Trans. A utomatic Control (Correspondence), vol. AC-11. pp. 311-312. April 1966.

[11] M. A. Aizerman and F. R. Gantmacher, Absolute Stability of Regulator Systems, San Francisco, Calif.: Holden-Das, 1964. 